F)

\title{
A Modular Software System for Three Patterns of Acetabular Deficiency Analysis
}

\author{
Linli Zheng ${ }^{1 *}$, Yangyang Lin $^{2 \dagger}$, Xiaoyan Zhang ${ }^{3 *}$, Qianhui Ling ${ }^{4 \S}$, a \\ Weiming Liao ${ }^{1 * *}$ and Peihui $\mathrm{Wu}^{1, \dagger \dagger}$ \\ ${ }^{1}$ Joint Surgery, First Affiliated Hospital of Sun Yat-sen University, Guangzhou, China \\ ${ }^{2}$ Rehabilitation Medicine, the Six Affiliated Hospital, Sun Yat-sen University, Guangzhou, China \\ ${ }^{3}$ College of Computer Science \& Software Engineering, Shenzhen University, Shenzhen, China. \\ ${ }^{4}$ Zhongshan Medical School, Sun Yat-sen University, Guangzhou, China. \\ alexandracefoxmail.com, linyy49@mail.sysu.edu.cn, \\ xyzhang15@szu. edu.cn, lingqh@outlook.com, 13809775039@163.com, \\ wupeihui@mail2.sysu.edu.cn
}

\begin{abstract}
In order to help orthopedists, evaluate the morphological characteristics of the acetabulum of patients with osteoarthritis, a 3-dimensional (3D) acetabular morphologic parameters measurements software dedicated to the hip was developed. The system includes three modules: 1) Identify the anterior pelvic plane (APP) of the pelvis model; 2) Identify the circular rim of the acetabular wall; 3) Automatically and interactively measure the 3D morphological parameters of the dysplastic acetabulum. The automatic parameter measurement function of this software could fast and accurately measure the 3D morphological parameters of the dysplastic acetabulum. These automatically measured parameters were close to those measured manually with error generally less than $2 \mathrm{~mm}$. This software was used to measure acetabular morphological parameters in 61 patients. Two types of dysplastic acetabula were identified by the thickness of the medial wall on the lower margin of the acetabulum Tb: type I was a thin acetabulum (35 cases, $\mathrm{Tb} \leq 10.0 \mathrm{~mm}$ ) and type II was a thick acetabulum (26 cases, $\mathrm{Tb}>10.0 \mathrm{~mm})$. the result of the acetabular morphological characteristic analysis, it can be found that the thickness of the medial wall is an important morphological characteristic for the THA preoperative surgical planning, and the thickened medial wall could be a misleading factor for the suboptimal placement of the cup.
\end{abstract}

\footnotetext{
* Designed and perform the study, do the statistical analysis and wrote the manuscript

${ }^{\dagger}$ Revised the draft

\$ Provided the software for morphological analysis

${ }^{\S}$ Collect clinical data and do statistical analysis

${ }^{* *}$ Reviewed and revised the draft

${ }^{\dagger}$ Designed the study, provided the software, wrote, revised and edited the manuscript
} 


\section{Introduction}

Developmental dysplasia of the hip (DDH) is the most common cause of secondary osteoarthritis (OA) in young adults (Rhodes \& Clarke, 2014). Acetabular deformities and insufficient bone stock increase the difficulty of achieving balance in restoration of the anatomical hip center, accuracy of cup orientation, and sufficient host bone-cup coverage (Fukui, Kaneuji, Sugimori, Ichiseki, \& Matsumoto, 2013) (Nishii, Sakai, Takao, \& Sugano, 2015) (Li, Mao, Oni, Dai, \& Zhu, 2013). When planning hip surgery for patients with DDH, accurate assessment of the quantitative parameters of the acetabulum is essential, as the 3-dimensional (3D) morphological abnormalities of the acetabulum are closely associated with the occurrence of disease in the hip joint (Ma, et al., 2014).

Therefore, it is necessary to establish a standardized, simple and feasible 3D morphological measurement method of acetabulum to meet the needs of clinical application. In order to efficiently and accurately measure the morphological characteristic of the acetabulum of patients with osteoarthritis of the hip, a modular software was developed. It includes three modules: 1) Identify the anterior pelvic plane (APP) of the pelvis model; 2) Identify the circular rim of the acetabular wall; 3) Automatically and interactively measure the 3D morphological parameters of the dysplastic acetabulum.

\section{Method}

A hip-dedicated software which runs on Windows system and is written in $\mathrm{C}++$ language using Microsoft Visual Studio was developed. The development is based on Visualization Toolkit (VTK) and Microsoft Foundation Classes (MFC).

\subsection{Identify the APP of the pelvis model}

The 3D pelvis models are imported as STL files into the software for morphological measurements. The 3D pelvis models are reconstructed in Mimics. The APP of the pelvis model is defined based on the bilateral anterior superior iliac spines and the pubic tubercles. The vertical axis of the APP, the horizontal and sagittal planes are also determined. (Figure 1 a)

\section{2 . Identify the circular rim of the acetabular wall}

The acetabular outer rim circle is fitted in 3 steps. First, the acetabular outer rim plane is fitted using the least square method. Then, the acetabular outer rim plane is rotated to align with the XOY plane (normal vector is $(0,0,1)$ ) to project the $3 \mathrm{D}$ points on the circular rim to $2 \mathrm{D}$ points. Finally, the acetabular outer rim circle is fitted in the 2D plane and rotated back to the original 3D space (Figure 1 b).

\subsection{Automatic measurement of the 3D morphological parameters}

By generating the coronal and transaxial sections, the bone stock distribution was evaluated by the contour curve in each section (Figure $1 \mathrm{c}-\mathrm{i}$ ). Based on the thickness curve of bone stock distribution, the automatic measurement of the 3D morphological parameters of the dysplastic acetabulum includes the thickness of the acetabular dome $\left(\mathrm{T}_{\mathrm{d}}\right)$ and bottom $\left(\mathrm{T}_{\mathrm{b}}\right)$, height $\left(\mathrm{H}_{\mathrm{c}}\right)$, and width of the acetabulum $(\mathrm{W})$, height of the anterior $\left(\mathrm{H}_{1}\right)$ and posterior acetabular walls $\left(\mathrm{H}_{2}\right)$. (Figure $\left.1 \mathrm{j}-1\right)$ 


\subsection{Interactive measurement of the 3D morphological parameters}

The interactive measurement is designed to personalize the $3 \mathrm{D}$ morphological measurement of the dysplastic acetabulum.

\subsection{Evaluation}

In order to evaluate the accuracy of our system, the accuracy of automatic 3D morphological measurement was compared to manual measurement.

\subsection{D morphological parameters of the dysplastic acetabulum}

On each section, various morphological parameters were measured. Dysplastic acetabula could be classified into two groups based on the thickness of the medial wall: type I was a thin acetabulum (35 cases, $\mathrm{Tb} \leqslant 10.0 \mathrm{~mm}$ ) and type II was a thick acetabulum ( 26 cases, $\mathrm{Tb}>10.0 \mathrm{~mm}$ ).

\subsection{Statistical analysis}

The data from the different groups that had a normal distribution were compared using independent Student's t-tests. Stepwise linear regression was used to identify factors that correlated with cup malpositioning. Correlations between variables were determined using Pearson's correlation coefficient.

\section{Results}

\subsection{Accuracy analysis of automatic 3D morphological measurement}

There were no statistical differences between the automatical and manual measurements by Student's t-tests $(\mathrm{p}<0.01)$.

\subsection{Acetabular morphological characteristic analysis}

The comparisons of the acetabular morphologic parameters in the thin and thick acetabulum groups are shown in Table 1. The significant statistical difference of these acetabular morphologic parameters confirmed our observation that the anatomy parameters of the dysplastic acetabula with thick medial wall could be significantly different from that with the thin medial wall.

\section{Discussion}

A modular software dedicated to efficiently and accurately measure the 3D morphological parameters of the dysplastic acetabulum of patients with osteoarthritis of the hip was successfully developed.

The study demonstrated that the automatically measured 3D morphological parameters were close to those measured manually with error generally less than $2 \mathrm{~mm}$. The error was in within the clinically acceptable range that determined by the surgeon in the THA (Shapi, Sulaiman, Hasan, \& Kassim, 2011).

It is useful to measure the quantitative parameters of the dysplastic acetabula of patients with osteoarthritis of the hip to reveal the unique characteristics of the pathological morphologies, to guide the accurate positioning of the cup, and to provide personalized preoperative planning (Nie, Pei, Shen, Kang, \& Li, 2015). According to the result of the acetabular morphological characteristics, an important 
finding was that the acetabulum with thickened medial wall generally has thicker acetabular dome, wider acetabulum, higher anterior wall and more significant osteophyte located in anterior wall compared with those with the normal medial wall.

The thickened medial wall described by Hartofilakidis et al. in his classification system (Hartofilakidis, Stamos, Karachalios, Ioannidis, \& Zacharakis, 1996), is also named as the central osteophyte, covering the subchondral area of the cartilaginous surface of the joint (Altman \& Collins, 1980), was found in all the 325 dysplastic hips. While in our cohort of cases, 26 cases (42.6\%) were found significant thickened central osteophyte, featuring invisible of the foveal floor, shallow and homogeneous of the acetabulum. Having thickened medial wall could be a misleading factor for the suboptimal placement of the cup, particularly to the young, inexperienced surgeon.

The acetabular bone stock is a substantial factor with regard to the acetabular component placement. Type of bony deformity should be considered preoperatively, and hip rotation center reconstruction at a normal anatomic location must be balanced with the demanding of sufficient bone-acetabular component coverage.

One problem of using our software is that the direction of the coronal sections may need to be adjusted according to the acetabular character of patients. Another problem is that the direction of thickness measurement line directly affects the accuracy of thickness measurement.

\begin{tabular}{ccccc}
\hline \multirow{2}{*}{ Name } & \multirow{2}{*}{ Measurement } & \multicolumn{2}{c}{ Measurement } & \multirow{2}{*}{ P value } \\
\cline { 3 - 4 } & & Thin & Thick & \\
\hline $\mathrm{H}_{\mathrm{C}}$ & $47.6 \pm 10.8$ & $46.3 \pm 11.2$ & $49.4 \pm 10.3$ & 0.278 \\
$\mathrm{~T}_{\mathrm{d}}$ & $39.0 \pm 7.0$ & $38.0 \pm 6.6$ & $40.4 \pm 7.5$ & 0.199 \\
$\mathrm{~T}_{\mathrm{b}}$ & $9.7 \pm 5.6$ & $5.3 \pm 1.6$ & $15.7 \pm 2.8^{*}$ & $<0.001$ \\
$\mathrm{~W}$ & $50.8 \pm 5.9$ & $49.3 \pm 5.8$ & $52.7 \pm 5.7^{*}$ & $<0.05$ \\
$\mathrm{H}_{1}$ & $28.2 \pm 5.6$ & $26.8 \pm 4.6$ & $30.1 \pm 6.3^{*}$ & $<0.05$ \\
$\mathrm{H}_{2}$ & $38.8 \pm 5.3$ & $38.2 \pm 5.7$ & $39.6 \pm 4.6$ & 0.292 \\
\hline
\end{tabular}

Table 1 Morphology measurements of 61 hips with DDH in two groups (type I: Thin acetabulum, $\mathrm{Tb} \leq 10.0 \mathrm{~mm}$; Type II: Thick acetabulum, $\mathrm{Tb}>10.0 \mathrm{~mm})$. $\mathrm{P}$ value less than 0.05 is considered as statistical significance. ${ }^{*} \mathrm{P}$ value less than 0.05 is considered as statistical significance 

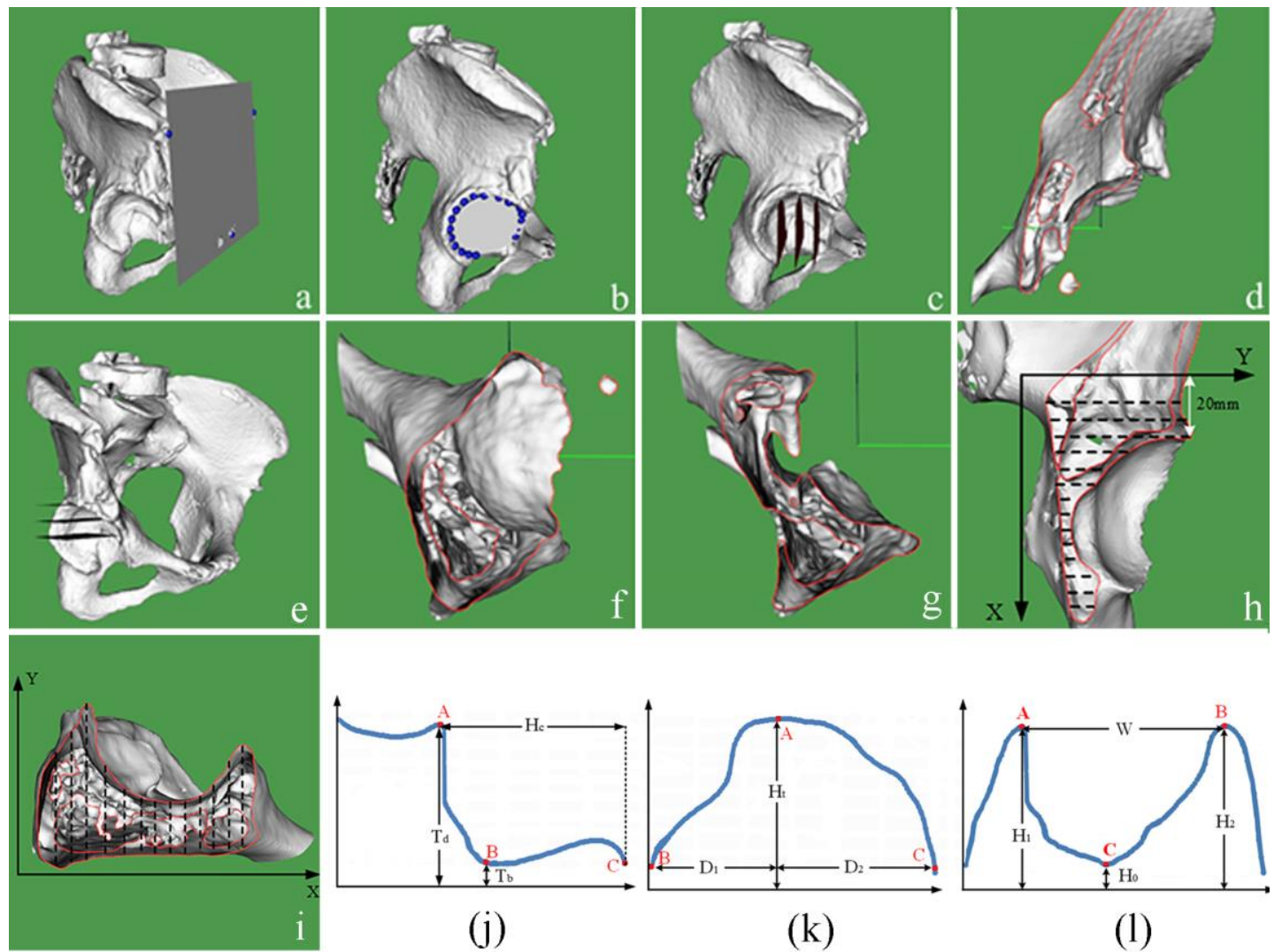

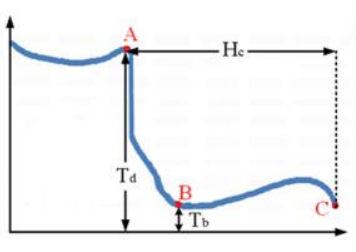

(j)

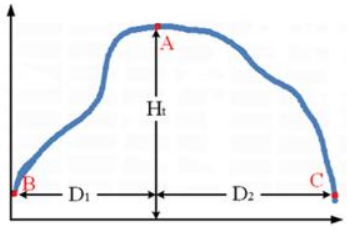

$(\mathrm{k})$

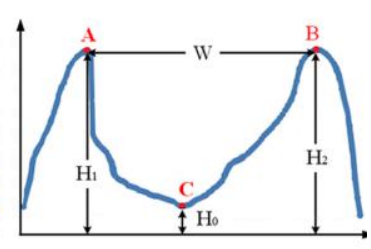

(1)

Figure 1 (a) The APP of the pelvis model. (b) The fitted circle of the acetabular wall. (c)-(d) Coronal sections. (e)-(g) Transaxial sections. (h) Thickness measurement in the coronal section. (i) Thickness measurement in the transaxial section. (j) The thickness curve of bone stock distribution in coronal sections. (k) The unimodal diagram of the superior transaxial section. (1) The bimodal diagram of the inferior and middle transaxial sections.

\section{References}

Altman, R., \& Collins, B. (1980). Musculoskeletal manifestations of Paget's disease of bone. . Arthritis Rheum, pp. 23:1121-1127.

Fukui, K., Kaneuji, A., Sugimori, T., Ichiseki, T., \& Matsumoto, T. (2013). How far above the true anatomic position can the acetabular cup be placed in total hip arthroplasty? . Hip Int, pp. 23:129-134.

Hartofilakidis, G., Stamos, K., Karachalios, T., Ioannidis, T., \& Zacharakis, N. (1996). Congenital hip disease in adults. Classification of acetabular deficiencies and operative treatment with acetabuloplasty combined with total hip arthroplasty. J Bone Joint Surg Am, pp. 78:683-692.

Li, H., Mao, Y., Oni, J., Dai, K., \& Zhu, Z. (2013). Total hip replacement for developmental dysplasia of the hip with more than $30 \%$ lateral uncoverage of uncemented acetabular components. . Bone Joint J, pp. 95-B:1178-1183. 
Ma, H., Han, Y., Yang, Q., Gong, Y., Hao, S., Li, Y., \& Liu, Y. (2014). Three-Dimensional Computed Tomography Reconstruction Measurements of Acetabulum in Chinese Adults. The Anatomical Record, pp. 297:643-649.

Nie, Y., Pei, F., Shen, B., Kang, P., \& Li, Z. (2015). Implication of acetabular width on the anteroposterior pelvic radiograph of patients with developmental dysplasia of the hip. $J$ Arthroplasty, pp. 30:489-494.

Nishii, T., Sakai, T., Takao, M., \& Sugano, N. (2015). Fluctuation of Cup OrientationDuring Press-Fit Insertion: A Possible Cause of Malpositioning. . J Arthroplasty, pp. 30:1847-1851.

Rhodes, A., \& Clarke, N. (2014). A review of environmental factors implicated in human developmental dysplasia of the hip. J Child Orthop, pp. 8:375-379.

Shapi, A., Sulaiman, R., Hasan, M., \& Kassim, A. (2011). An Automated Size Recognition Technique For Acetabular Implant in Total Hip Replacement. International Journal of Computer Science \& Information Technology (IJCSIT), p. 3. 